\title{
The expression of STEAP4 in peripheral blood predicts the outcome of septic patients
}

\author{
Haiyan Jiang ${ }^{1,2 \#}$, Yansong Dong ${ }^{2 \#}$, Dajun Yan $^{2}$, Yao Wu ${ }^{2}$, Yue Wang ${ }^{2}$, Yuting Ren ${ }^{2}$, Guomin Mao', \\ Guiwen Liang ${ }^{2}$, Wei Liu ${ }^{3}$, Yang Zhou ${ }^{3}$, Zhongwei Huang ${ }^{2}$, Lei Qi ${ }^{2,4}$ \\ ${ }^{1}$ Department of Health Medicine, Affiliated Hospital of Nantong University, Nantong, China; ${ }^{2}$ Department of Emergency Medicine, Affiliated \\ Hospital of Nantong University, Nantong, China; ${ }^{3}$ Department of Obstetrics and Gynecology, Affiliated Hospital of Nantong University, Nantong, \\ China; ${ }^{4}$ Rugao Branch (Rugao Bo'ai Hospital), Affiliated Hospital of Nantong University, Nantong, China \\ Contributions: (I) Conception and design: Z Huang, H Jiang; (II) Administrative support: L Qi, Y Dong; (III) Provision of study materials or patients: \\ Y Wu, Y Wang; (IV) Collection and assembly of data: Y Ren, G Mao; (V) Data analysis and interpretation: G Liang, W Liu; (VI) Manuscript writing: \\ All authors; (VII) Final approval of manuscript: All authors. \\ \#The authors contributed equally to this work. \\ Correspondence to: Lei Qi; Zhongwei Huang. Department of Emergency Medicine, Affiliated Hospital of Nantong University, 20 West Temple Road, \\ Nantong 226001, China. Email: qilei723@ntu.edu.cn; tdfyhuangzw@163.com.
}

\begin{abstract}
Background: Sepsis is a systemic disease characterized by extensive inflammatory responses and impaired organ function, which are characteristics that make it easily missed and complex to treat. A large number of laboratory and clinical studies on the diagnosis and treatment of sepsis have been continuously carried out, confirming the importance of mitochondrial function during the development of sepsis. STEAP4 is an important metalloreductase in mitochondria, which is involved in the biogenesis and respiratory chain of mitochondria. The role of STEAP4 in inflammation remains controversial. Research in this field may contribute to the development of new diagnostic and treatment options for sepsis.

Methods: The expression of STEAP4 was measured in the peripheral blood of patients with severe sepsis and compared with healthy controls. Cell and mouse inflammatory models were established to detect the expression of STEAP4 and other inflammatory cytokines.

Results: (I) The expression of STEAP4 in the peripheral blood of patients with severe sepsis is higher than that of healthy volunteers $(\mathrm{P}<0.01)$, which is related to the SOFA score and transaminase. (II) STEAP4 has a certain predictive effect on the outcome of patients [area under curve (AUC) $=0.696, \mathrm{P}<0.05,95 \%$ CI: 0.528 to 0.833 ]. (III) Inflammation led to increased expression of STEAP4 gene in RAW264.7 cells and mouse liver tissue.
\end{abstract}

Conclusions: The expression of STEAP4 is elevated in the early stage of sepsis and the degree of its elevation can be used to predict the clinical outcome of sepsis patients.

Keywords: STEAP4; sepsis; biomarker

Submitted Apr 14, 2021. Accepted for publication Oct 22, 2021.

doi: 10.21037/atm-21-2794

View this article at: https://dx.doi.org/10.21037/atm-21-2794

\section{Introduction}

Sepsis is a systemic inflammatory reaction accompanied by multiple organ failure, and involves important pathological processes such as inflammatory responses, energy metabolism disorder, and cell and tissue damage, among others $(1,2)$. The rapid occurrence of organ failure and shock lead to high mortality. The mechanism of organ dysfunction caused by excessive inflammation is still uncertain (3). The morphological changes found in mitochondria suggest that the mitochondrial energy crisis may be related to organ dysfunction. During sepsis, many 
mitochondrial functions are altered, including impaired use of metabolic substrates, increased reactive oxygen species production, decreased autophagy in damaged mitochondria, and changes in mitochondrial biogenesis and kinetics. Recent studies have found that six-transmembrane epithelial antigen of the prostate 4 (STEAP4; also known as TIARP and $S T A M P 2$ ), a mitochondrial metalloproteinase, plays an important role in inflammatory and metabolic diseases (4), and its feature of linking inflammation and metabolism has attracted our great interest.

STEAP4 protien is able to maintain iron and copper homeostasis by reducing $\mathrm{Fe}^{3+}$ to $\mathrm{Fe}^{2+}$ and $\mathrm{Cu}^{2+}$ to $\mathrm{Cu}^{+}$and promoting their transmembrane transport (5), plays an important role in the inflammatory response of cells. It was found to be highly expressed after the induction of TNF- $\alpha$ (6), and was later proven to be upregulated by other inflammatory cytokines such as IL-6 and IL-1 $\beta$ (7). A large number of studies have confirmed that STEAP4 is involved in the chronic inflammatory response of adipocytes during the pathogenesis of diabetes $(7,8)$, and lower STEAP4 expression is associated with obesity and insulin resistance (9). Furthermore, some studies have shown that STEAP4 plays a protective role against inflammatory damage in hepatocytes (10), mesangial cells (11), and synovial cells (12). These studies indicate that STEAP4 plays a protective role in the development of inflammatory diseases and metabolic disorders, which suggests a role for STEAP4 in sepsis. However, the role of STEAP4 in the process of sepsis, a serious infectious disease, is still unknown, we hypothesized that STEAP4 may alter the severity of sepsis by regulating mitochondrial energy metabolism, which is of great value for the clinical diagnosis and treatment of sepsis, and our study aimed to find an association between STEAP4 and sepsis.

In the present study, we assessed the predictive value of STEAP4 and unexpectedly found a certain relationship with septic hepatocyte injury. We also tested our hypothesis in a simple cellular and mouse model. We present the following article in accordance with the STARD reporting checklist (available at https://dx.doi.org/10.21037/atm-21-2794).

\section{Methods}

\section{Study population and definition}

In this prospective study, we recruited 39 non-surgical and non-traumatic adult patients with sepsis for over 1 year from January to December 2020 at the Affiliated Hospital of Nantong University. For comparison, we enrolled 30 sex- and age-matched healthy individuals without infection as the control group. Patients were monitored every day, and sepsis or septic shock was diagnosed according to the sepsis criteria defined by the Third International Consensus Definitions published in 2016 (1). All patients enrolled in this study met the diagnostic criteria of sequential organ failure assessment (SOFA) score $\geq 2$ points higher than those associated with baseline status. Exclusion criteria included patients with (I) hematological disease and those receiving chemotherapy, (II) simultaneous comorbidities (such as combined tumors, chronic inflammation, and metabolic disease, including obese patients) that may have affected results, (III) acute or chronic liver disease, and (IV) patients admitted 28 days prior. The process is shown in Figure 1. All procedures performed in this study involving human participants were in accordance with the Declaration of Helsinki (as revised in 2013). The study was approved by Ethics Committee of the Affiliated Hospital of Nantong University (No. 2017-L021) and informed consent was taken from all the patients.

\section{Clinical assessment and therapy}

A standardized assessment scale was used to record clinical severity indicators including the Acute Physiology and Chronic Health Assessment II (APACHE II) score and SOFA score based on the worst physiological parameters recorded within 24 hours after being admitted to the hospital. Records included vasoactive substances, ventilator support, markers of organ damage, and other treatment information. Once patients are diagnosed with sepsis, they are treated according to the 2016 guidelines, including blood volume replenishment, treatment of hypoxia, antiinfection, organ function support, and stabilization of the internal environment.

\section{Outcome determination}

The endpoint of follow-up was 28 days after admission or death.

\section{Collection of blood samples}

Peripheral blood was collected for RNA analysis and STEAP4 detection, which was performed within 24 hours of the patient's admission. 


\section{Clinical biochemical detection}

Examinations were carried out in the hospital laboratory with accepted methods. Clinical parameters including white blood cells, C-reactive protein (CRP), alanine aminotransferase (ALT), and aspartate aminotransferase (AST) were tested at the time of admission, and subsequent tests were determined by the doctor according to the condition of patients. CRP level was determined by enzyme immunoassay, procalcitonin level by enzymelinked fluorescence assay, and lactic acid level by serum assay. Other parameters, such as white blood cell counts and differential counts, as well as creatinine (Cr), glutamate transaminase (GOT), and hemoglobin ( $\mathrm{Hb}$ ) levels, were also measured using internationally recognized laboratory methods.

\section{Cell culture and treatment}

The mouse macrophage RAW264.7 cells used in this study were purchased from Shanghai Cell Bank of Chinese Academy of Sciences and cultured in complete medium containing $10 \%$ fetal bovine serum. Lipopolysaccharide (LPS, purchased from Sigema, USA) was used to model sepsis cells at a dose of $100 \mathrm{ng} / \mathrm{mL}$.

\section{Cecal ligation and perforation (CLP)}

All animal experiments were undertaken in accordance with the National Institute of Health Guide for the Care and Use of Laboratory Animals. Animal experiments were approved by Ethics Committee of Affiliated Hospital of Nantong University. Cervical dislocation was used to provide mice with a fast and painless death. The operation was carried out according to the protocol published by Rittirsch et al. (13). Isoflurane was used as an inhalation anesthetic to reduce the interference of anesthesia on the experiment and improve the success rate of the operation.

\section{$R N A$ extraction and real-time quantitative polymerase chain reaction ( $q R T-P C R)$}

Total RNA was extracted using TRIzol reagent (Invitrogen, USA), then reverse transcription was performed using HiScript II Q Select RT SuperMix for qPCR (+gDNA wiper) (Vazyme, Nanjing, China), according to the following procedure: $1 \mu \mathrm{g}$ of total RNA and $4 \times \mathrm{g}$ DNA Wiper

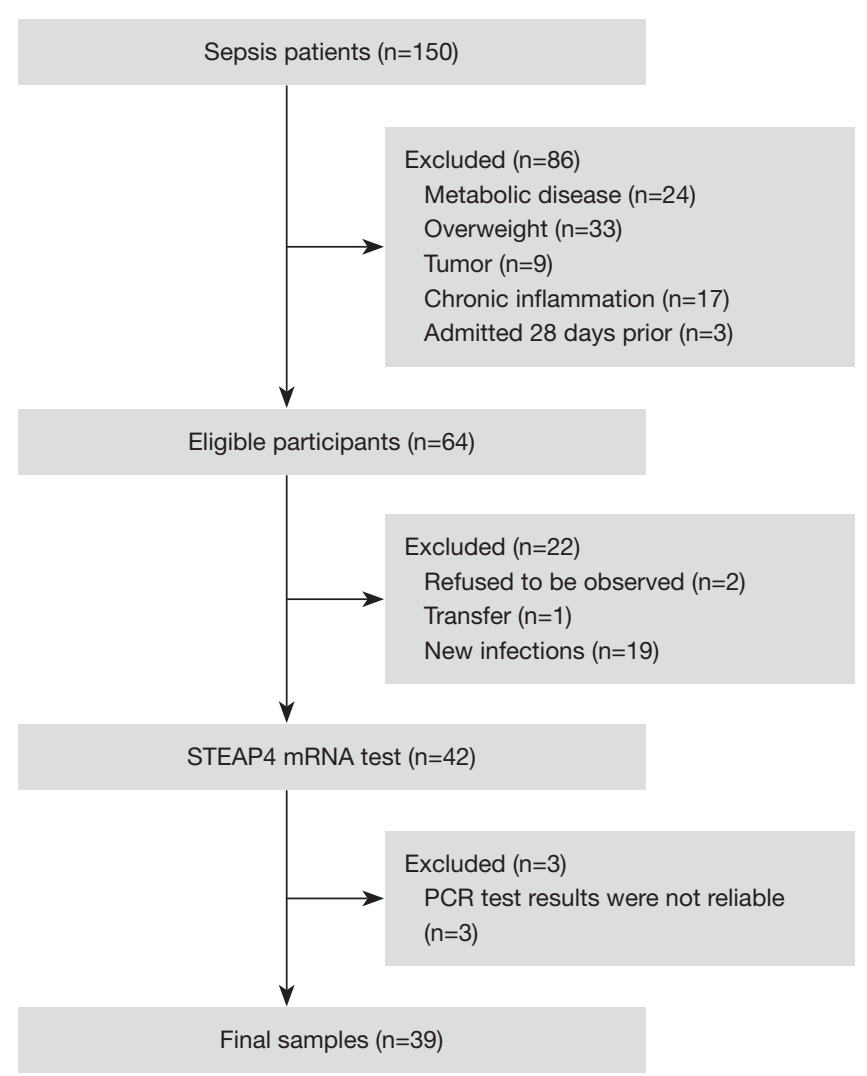

Figure 1 Procedures for clinical trials. STEAP4, six-transmembrane epithelial antigen of the prostate 4 .

mix was incubated at $42{ }^{\circ} \mathrm{C}$ for $2 \mathrm{~min}$ to remove genome contamination, then $5 \times$ HiScript II QRT SuperMix was added to the reaction mixture and incubated at $37{ }^{\circ} \mathrm{C}$ for $15 \mathrm{~min}$ and $85^{\circ} \mathrm{C}$ for $5 \mathrm{~s}$. The resulting cDNA was used for real-time PCR detection using the Step One Plus real-time PCR system (Applied Biosystems, Waltham, MA, USA) with ChamQ Universal SYBR qPCR Master Mix (Vazyme, Nanjing, China). The conditions for PCR were $95^{\circ} \mathrm{C}$ for $30 \mathrm{~s}$, followed by 35 cycles of $95{ }^{\circ} \mathrm{C}$ for $5 \mathrm{~s}$ and $63{ }^{\circ} \mathrm{C}$ for $30 \mathrm{~s}$. The housekeeping gene GAPDH was used as the reference gene. The relative expression of the genes of interest was normalized to the geometric mean of GAPDH expression.

The sequences of the primers used are shown in Table 1.

\section{Western blot}

Protein analysis was performed as previously described (14). Antibodies against STEAP4 (Cat No. 11944-1-AP) were purchased from Proteintech. 
Table 1 Primer sequences for qRT-PCR

\begin{tabular}{llc}
\hline Gene & Forward primer (5'-3') & Reverse primer (5'-3') \\
\hline STEAP4 (homo) & GAAACTTCCCTCTACCCGCC & ACAGTTCTTGAGACCTGAGGG \\
STEAP4 (mouse) & ACCTCCCTGGTATTCTCGCT & AGGGCCTGAGTAATGGTTGC \\
IL-1 $\beta$ & AGCCATGGCAGAAGTACCTG & TGAAGCCCTTGCTGTAGTGG \\
IL-6 & TCCGGAGAGGAGACTTCACA & CATAACGCACTAGGTTTGCCG \\
GAPDH (homo) & AATGGGCAGCCGTTAGGAAA & GCGCCCAATACGACCAAATC \\
GAPDH (mouse) & CAGGTTGTCTCCTGCGACTT & TATGGGGGTCTGGGATGGAA
\end{tabular}

STEAP4, six-transmembrane epithelial antigen of the prostate 4; GAPDH, glyceraldehyde-3-phosphate dehydrogenase; IL-1 $\beta$, Interleukin $1 \beta$; IL-6, Interleukin 6.

\section{Statistical analysis}

IBM SPSS Statistics 20, GraphPad Prism 8, Medclac application was used for data analysis. The differences between the two groups of normal distribution data were verified by the independent $t$-test, the classified data were analyzed by the chi-square test, and the Kaplan-Meier test was used to verify the differences in the survival curve. $\mathrm{P}<0.05$ was considered to have significant differences between the two groups of data.

\section{Results}

The expression of STEAP4 in the peripheral blood of bealthy participants is lower than that in sepsis patients

The general information shows that there was no significant difference in the basic characteristics of patients with sepsis from that of the healthy people as shown in Table 2 . To explore whether the STEAP4 gene changes in sepsis, we obtained the expression of STEAP4 mRNA from the peripheral blood of 30 healthy people and 39 septic patients by q-RT PCR. It turned out that in healthy people the expression of STEAP4 mRNA is lower than that in patients with sepsis $(1.14 \pm 0.15$ vs. $3.21 \pm 0.51, \mathrm{P}=0.0008$, Figure 2$)$.

\section{High STEAP4 expression is associated with acute hepatocyte injury}

The clinical data of patients with sepsis were analyzed, in which the expression level of the STEAP4 mRNA was linearly correlated with ALT $(\mathrm{r}=0.6584, \mathrm{P}<0.01$, Figure $3 A)$ and AST ( $r=0.5236, \mathrm{P}<0.01$, Figure $3 B$ ) levels in peripheral blood. The relationship between STEAP4 and ALT and
AST suggests that STEAP4 may be associated with septic liver injury. Patients were divided into an acute hepatocyte injury (AHI) group and a non-acute hepatocyte injury (non-AHI) group based on whether ALT and AST values exceed the upper limit of normal values. Comparing the two groups, the expression of STEAP4 was higher in the ALI group than in the non-ALI group $(\mathrm{P}=0.047$, Figure $3 C)$ while there was no difference in baseline data (Table 3).

\section{STEAP4 has certain predictive power for the outcome of patients with sepsis}

There was a linear correlation between the expression of STEAP 4 and SOFA score $(\mathrm{r}=0.546, \mathrm{P}=0.003$, Figure $4 A$ ) rather than the APACHE II score $(\mathrm{r}=0.136$, $\mathrm{P}=0.41$, Figure $4 B$ ), which indicates that STEAP4 may be associated with the severity and prognosis of sepsis. A receiver operating characteristic (ROC) curve was used to determine the potential ability of STEAP4 to predict the outcome of patients with sepsis, and the results are shown in Figure 5A, the area under curve (AUC) is 0.696 , $\mathrm{P}=0.036$, cutoff value $=1.6,95 \% \mathrm{CI}: 0.528$ to 0.833 . For further study, STEAP4 expression was used as the grouping criterion, it was found that the mortality rate of the patients in the high expression group was 10/24, which in the low expression group was $2 / 15$, and the survival analysis showed no significant difference in the 28 -day survival rate (Kaplan-Meier $\mathrm{P}=0.083$, Figure $5 B$ ) as well as the chi-square test $(\mathrm{P}=0.062)$. We believe that this non-difference is mainly due to the small sample size and the heterogeneity of the primary disease, so we validated the clinical findings in cell and animal experiments. 
Table 2 General characteristics of the participants

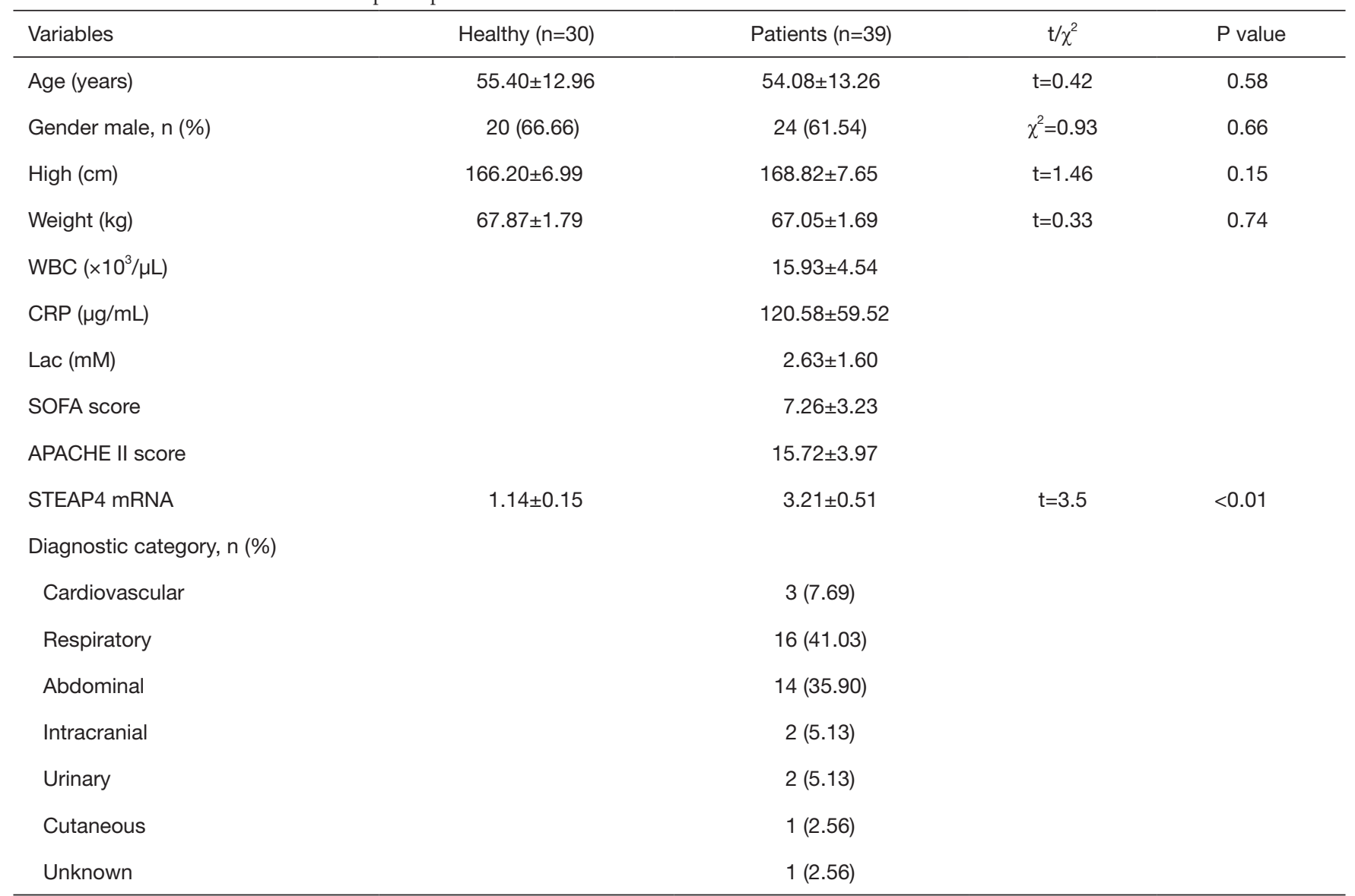

The data are shown as $\mathrm{n}(\%)$ or mean \pm SD. WBC, white blood cell count; CRP, C-reactive protein; Lac, lactic acid; SOFA, Sequential Organ Failure Assessment; APACHE II, Acute Physiology and Chronic Health Assessment II; STEAP4, six-transmembrane epithelial antigen of the prostate 4 .

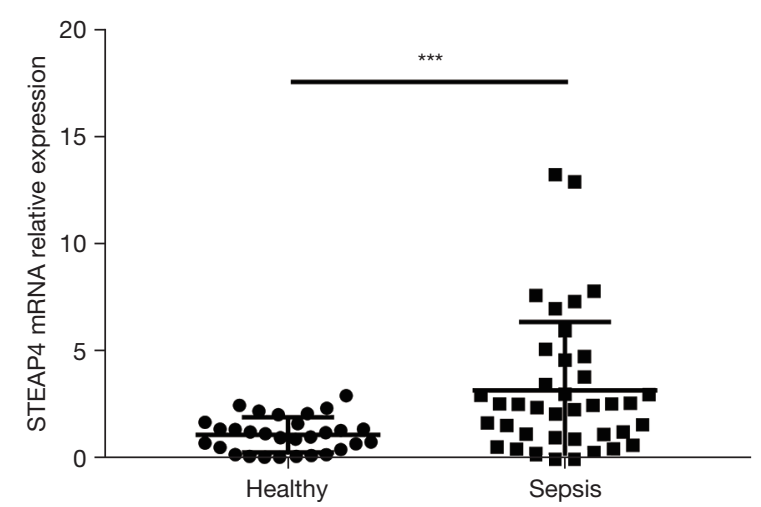

Figure 2 The expression of STEAP4 mRNA in the peripheral blood of sepsis patients was higher than that of healthy people $(n=39$ vs. $\mathrm{n}=30, \mathrm{P}=0.0008)$. ${ }^{* * *}, \mathrm{P}<0.01$. STEAP4, six-transmembrane epithelial antigen of the prostate 4 .
In addition, lactic acid levels are considered to be the most powerful indicator of clinical outcomes in patients with sepsis. When the lactic acid was used to assess the outcome of the patients with sepsis, the results showed that AUC $=0.759, \mathrm{P}=0.004$, cutoff value $=2,95 \%$ CI: $0.596-0.881$ (Figure 6).

\section{STEAP4 expression was significantly increased in LPS- induced RAW264.7 cells}

To further verify our clinical findings, RAW264.7 cells were treated with LPS to detect STEAP4 expression in sepsisinfected hepatocytes. As shown in Figure 7, both mRNA and protein expression of STEAP4 were significantly increased in septic liver cells. 

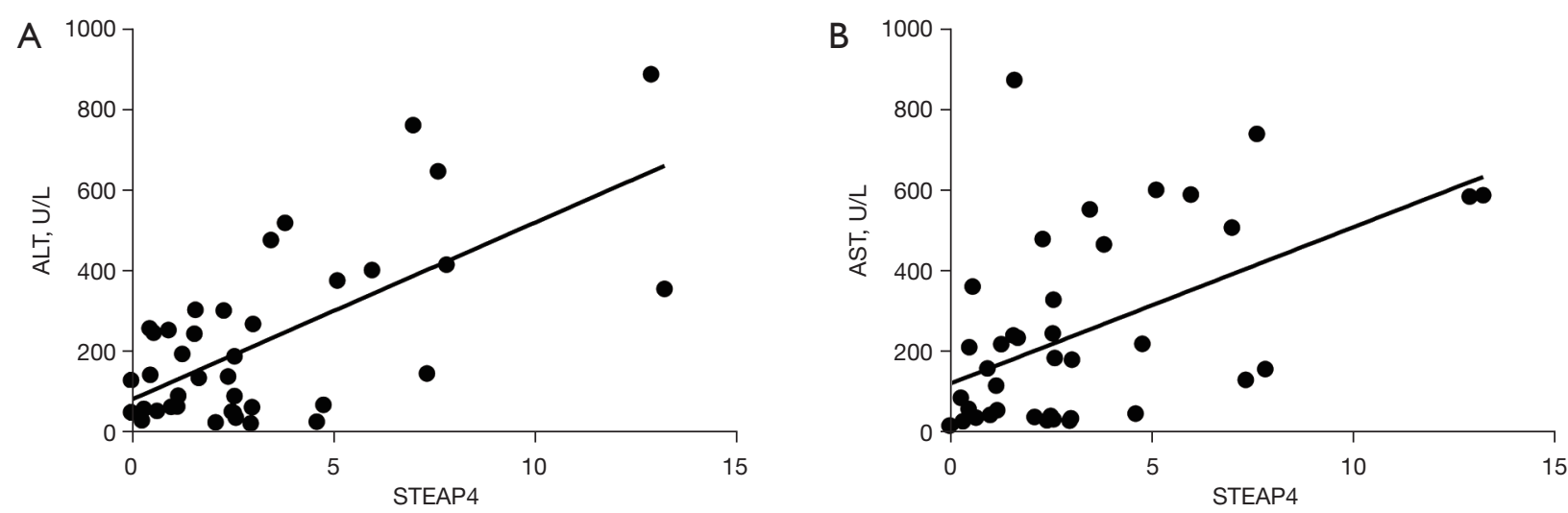

C

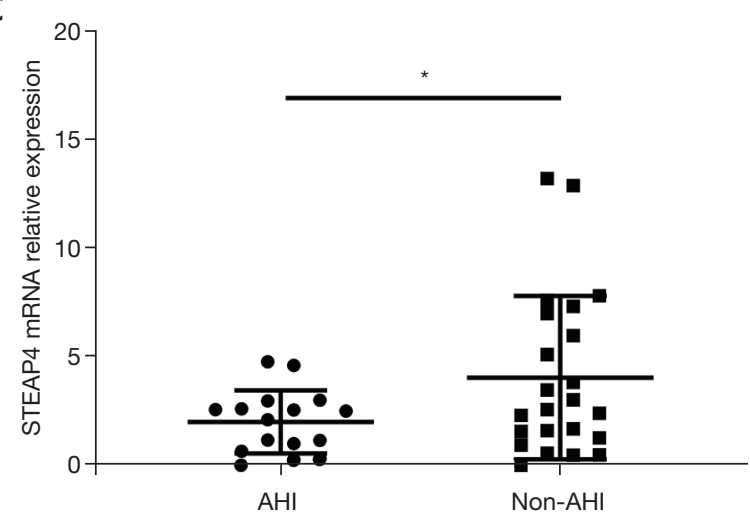

Figure 3 Correlation between STEAP4 and acute hepatocyte injury. (A) There was a linear correlation between STEAP4 expression and ALT level in the peripheral blood of patients $(\mathrm{r}=0.6584, \mathrm{P}<0.01)$; (B) The linear correlation between STEAP4 expression and AST level in the peripheral blood of patients was well established $(\mathrm{r}=0.5236, \mathrm{P}<0.01)$; (C) STEAP4 expression was higher in patients with hepatocyte injury than in patients without hepatocyte injury ( $\mathrm{n}=25$ vs. $\mathrm{n}=14, \mathrm{P}=0.047)$. *, $\mathrm{P}<0.05$. STEAP4, six-transmembrane epithelial antigen of the prostate 4; AHI, acute hepatocyte injury; ALT, alanine aminotransferase; AST, aspartate aminotransferase.

\section{The expression of STEAP4 was also increased in the livers of CLP mice}

The mRNA and protein expression of STEAP4 were measured in CLP-treated mouse liver tissues. As shown in Figure 8, the expression of STEAP4 in the liver tissue of sepsis mice was significantly higher than that in the nonsurgery group.

\section{Discussion}

For the first time, our study found that the expression of STEAP4 in the peripheral blood of sepsis patients was higher than that of healthy individuals, suggesting that STEAP4 plays a role in the process of sepsis.
This phenomenon is similar to the findings of most studies demonstrating that STEAP4 is elevated in many inflammatory diseases such as rheumatoid arthritis (15), pustular skin disorders (16), and adipocyte inflammation (17). In our study, it was unexpectedly found that the high expression of STEAP4 in the peripheral blood was positively correlated with the degree of elevated liver enzymes (ALT and AST), ROC analysis suggests that STEAP4 has a certain predictive ability for the outcome of sepsis, and may be used to diagnose the severity of sepsis. However, because of the sample size, further verification is needed to confirm this. Our cell and animal experiments demonstrated that sepsis led to the elevation of STEAP4 expression in liver. The question as to whether STEAP4 plays a role as a protective or harmful factor in sepsis has not yet been studied. 
Table 3 General characteristics of the patients

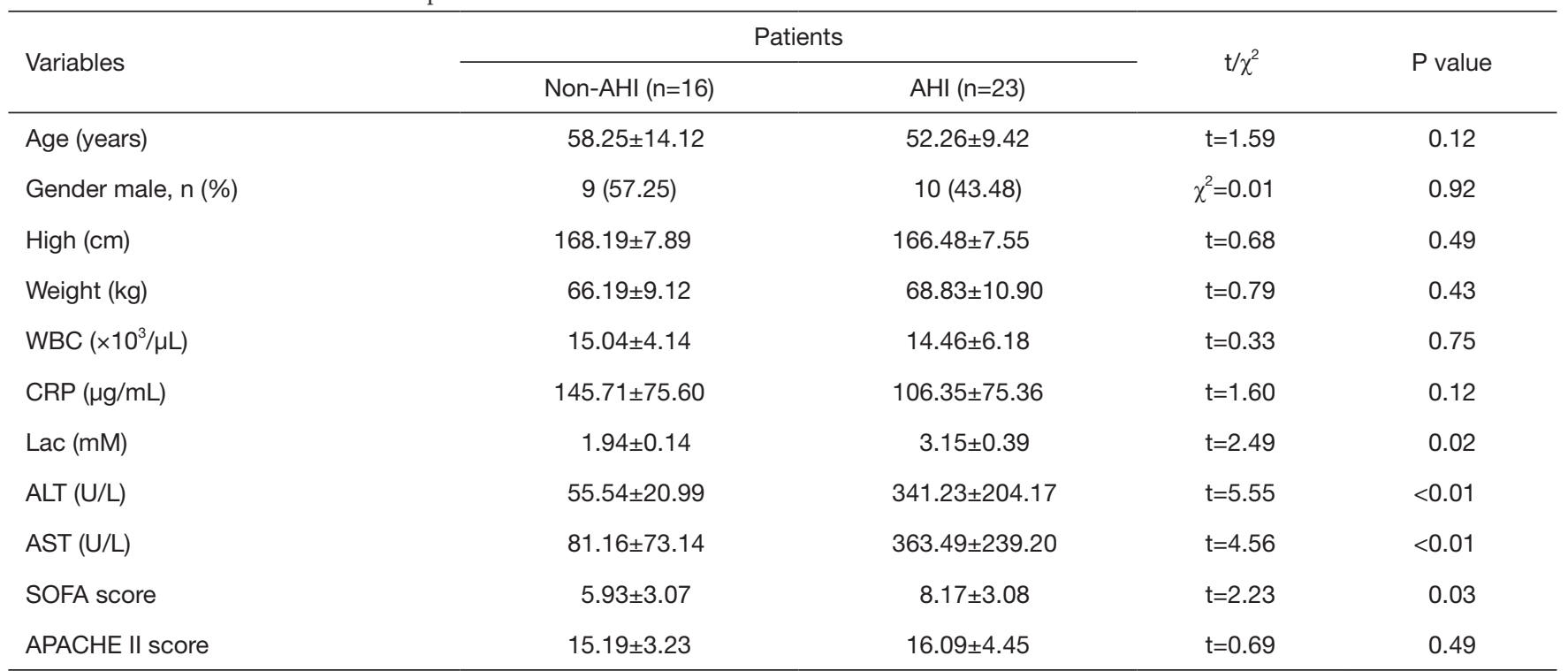

The data are shown as $\mathrm{n}(\%)$ or mean \pm SD. WBC, white blood cell count; CRP, C-reactive protein; Lac, lactic acid; SOFA, Sequential Organ Failure Assessment; APACHE II, Acute Physiology and Chronic Health Assessment II; STEAP4, six-transmembrane epithelial antigen of the prostate 4 .
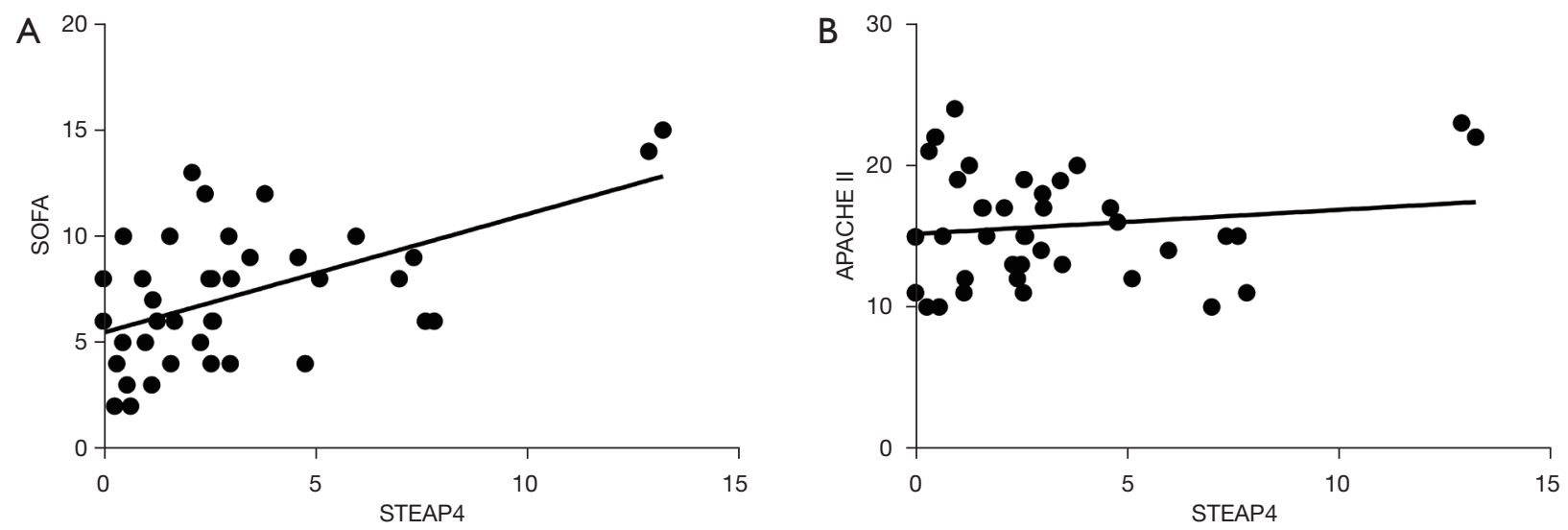

Figure 4 Correlation between STEAP4 and the severity of sepsis. (A) There was a linear correlation between STEAP4 expression and SOFA scores in the peripheral blood of patients $(\mathrm{r}=0.546, \mathrm{P}=0.003)$; (B) there was no linear correlation between STEAP4 expression and APACHE II scores in the peripheral blood of patients $(r=0.136, \mathrm{P}=0.41)$. STEAP4, six-transmembrane epithelial antigen of the prostate 4; SOFA, Sequential Organ Failure Assessment; APACHE, Acute Physiology and Chronic Health Assessment.

Furthermore, research results in related fields conflict with each other. Some researchers have pointed out that inflammatory cytokines increase the uptake of copper by cells by inducing STEAP4, activating NF-KB, and inhibiting caspase 3 (18). Another study also revealed that low expression of STEAP4 led to the increase of caspase 3 (19), suggesting that STEAP4 may increase the ability of cells to adapt to inflammatory stimulation by regulating the expression of apoptosis proteins. Also, studies have observed that an LPS-induced increase in the expression of STEAP4 in piglet liver reduces inflammation, oxidative stress, and inhibits the phagosome pathway (20). In contrast, some researchers 

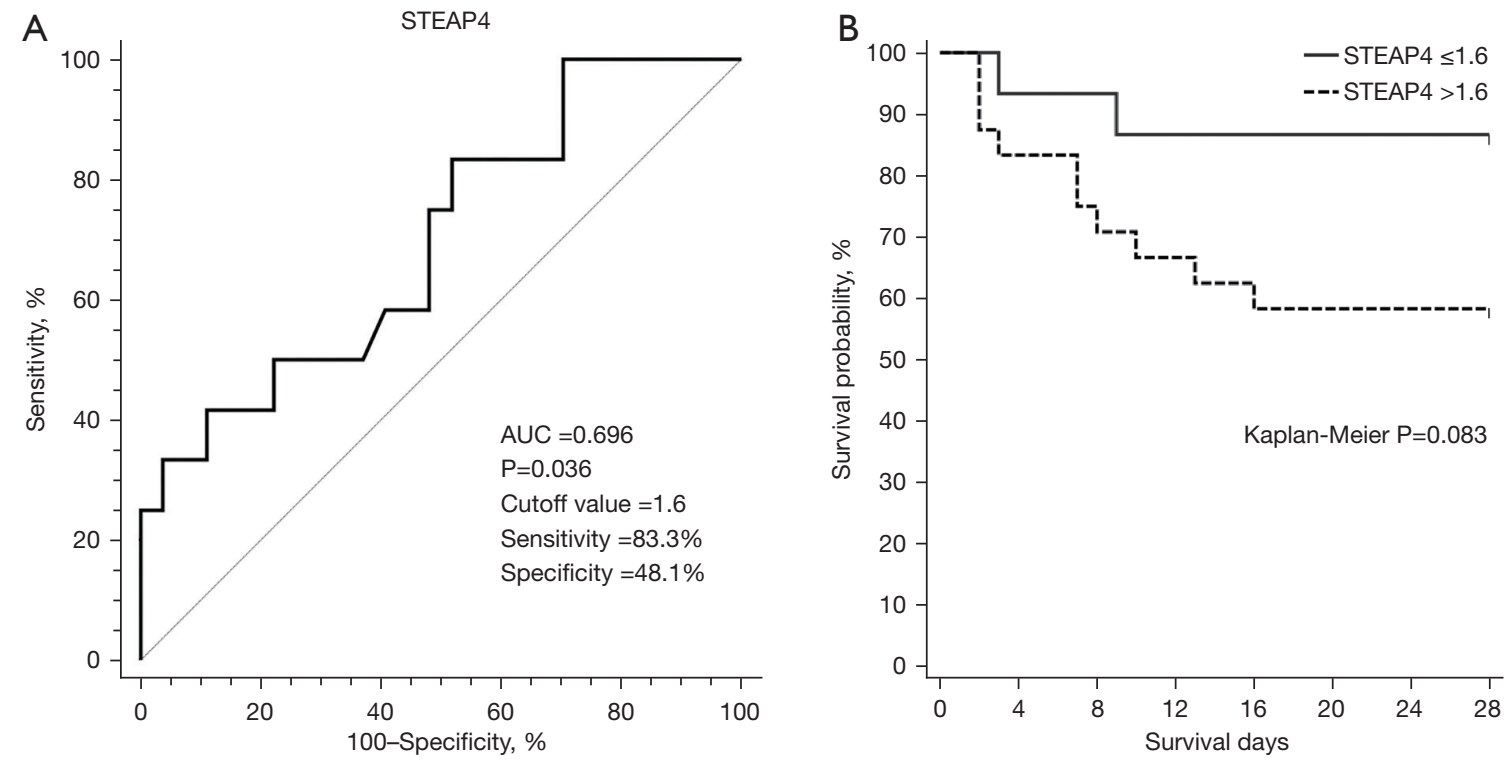

Figure 5 The ability of STEAP4 to predict the prognosis of sepsis. (A) The ability of STEAP4 to predict whether sepsis patients will die (AUC $=0.696, \mathrm{P}<0.05,95 \% \mathrm{CI}: 0.528$ to 0.833 ); (B) patients were divided into two groups according to whether STEAP4 expression was greater than 1.6, and the survival interval between the two groups was no difference (Kaplan-Meier P=0.083). STEAP4, six-transmembrane epithelial antigen of the prostate 4; AUC, area under curve.

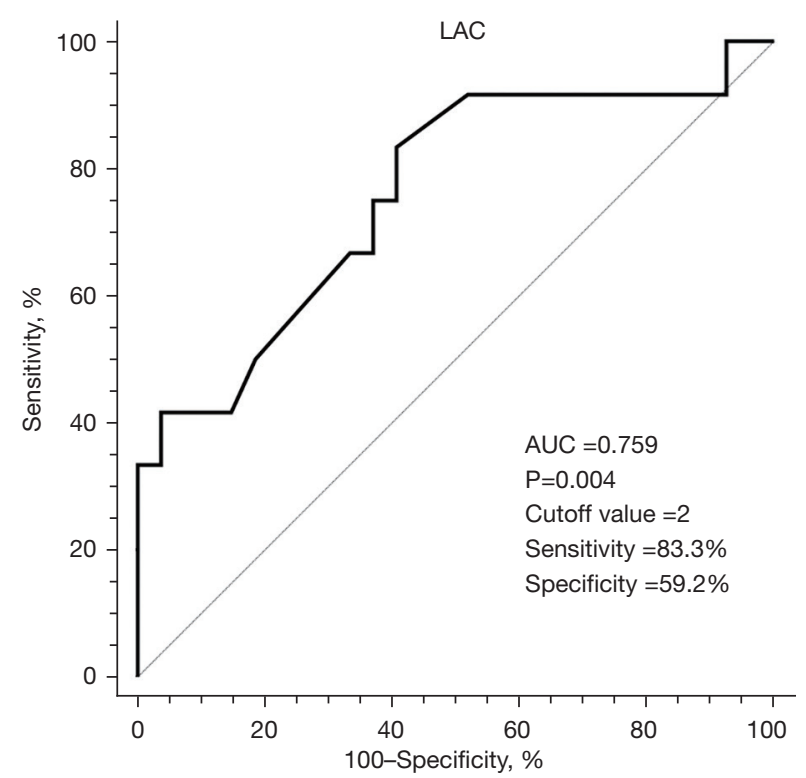

Figure 6 The ability of LAC to predict whether sepsis patients will die (AUC $=0.759, \mathrm{P}=0.004,95 \% \mathrm{CI}$ : 0.596-0.881). LAC, lactic acid; AUC, area under curve. believe that STEAP4 leads to the adverse outcomes of inflammation. The increase of STEAP4 in the lung tissue of mice with inflammation-induced pneumonia accelerated the copper-mediated cytotoxicity (21), while the overexpression of STEAP4 in the mouse colon led to mitochondrial iron balance. Increased production and disorders of reactive oxygen species occur in inflammation (22). These differences suggest that the expression changes of STEAP4 in sepsis are of research significance.

STEAP4 protein is widely present in mitochondria and cell membranes of various cells including macrophages which initiates the host's immune response through the recognition and binding of microbial components. Our study found that the expression of STEAP4 was significantly increased in mouse mononuclear macrophages stimulated by LPS, suggesting that STEAP4 may play a regulatory role in macrophage-mediated immune response. In addition, pathogen and antigen-antibody complexes were phagocytic and decomposed by monocyte macrophages or Kuffer cells in the liver, which not only does not conflict 

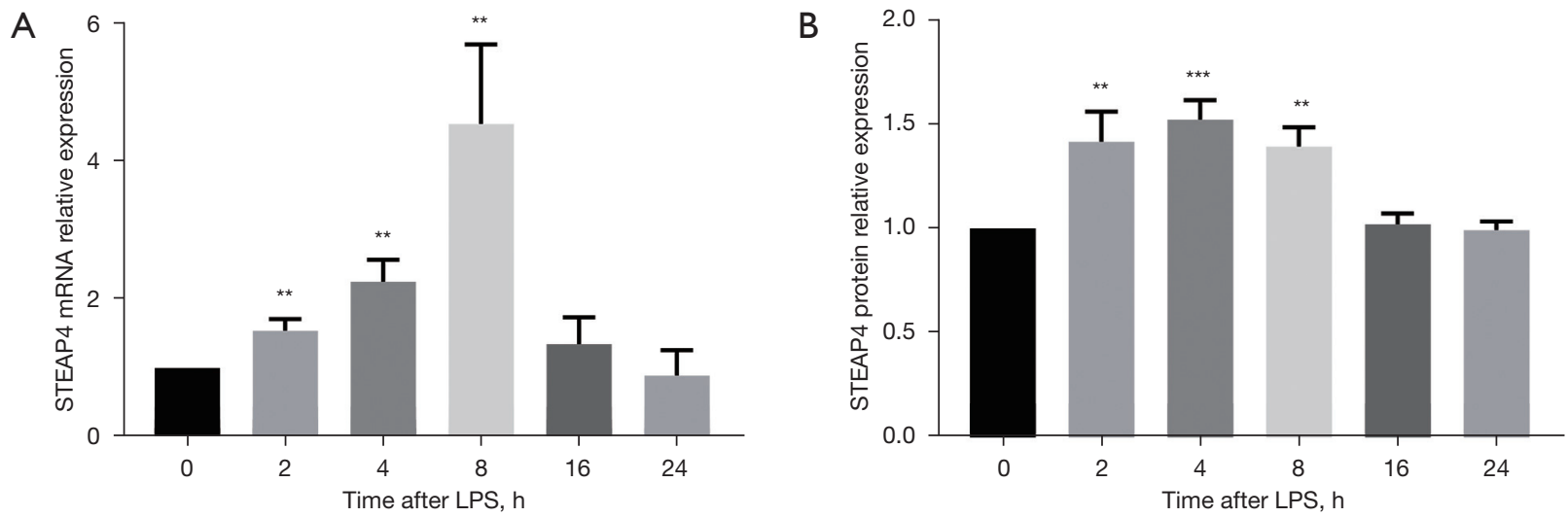

C

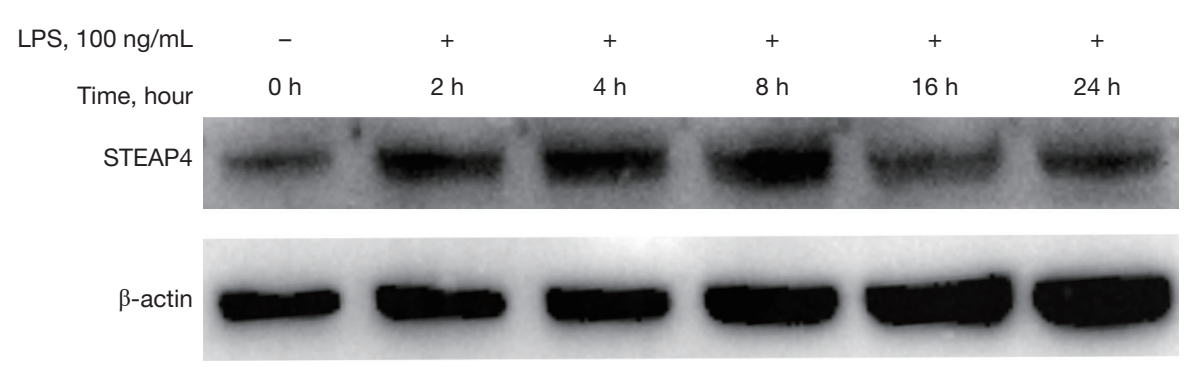

Figure 7 Changes of STEAP4 in LPS-treated RAW264.7 cells. (A) The transcriptional expression of STEAP4 in RAW264.7 cells firstly increased and then decreased after LPS stimulation, and reached a peak at 8 hours; (B) WB analysis showed that the expression of STEAP4 in LO2 cells was increased after LPS stimulation; (C) the grey level of STEAP4 protein expression in LO2 cells after LPS stimulation was detected by WB. **, $\mathrm{P}<0.02$; ***, $\mathrm{P}<0.01$. LPS, lipopolysaccharide.

with our cell experiments, but also suggests that the correlation between STEAP4 and hepatocyte damage may reflect septic liver damage due to the disordered host innate immune response. Septic state causes destruction of redox balance in many cell types, for this kind of situation, now we can do is to try to correct hypoxia, however, this method can increase organization's ability to absorb oxygen rather than the ability to use oxygen, STEAP4 gene involved in the regulation of mitochondrial oxidative respiration characteristics may make it becomes the key to maintain the redox balance.

There are some shortcomings in our study. The expression of STEAP4 was obtained from whole blood rather than a specific type of isolated cells, making it impossible to determine which kind of cells play a major role in the change in STEAP4 expression. Furthermore, the process of sepsis involves a variety of cells and organs (23), which cannot be represented by the function of a certain type of cells. Taking whole blood as the object of study can reveal the clinical characteristics of sepsis as macroscopically as possible, and is beneficial for the clinical application of the experimental results.

\section{Conclusions}

The high expression of STEAP4 in the peripheral blood of sepsis patients has certain diagnostic efficacy for the severity of the disease and the outcome of the patients. 

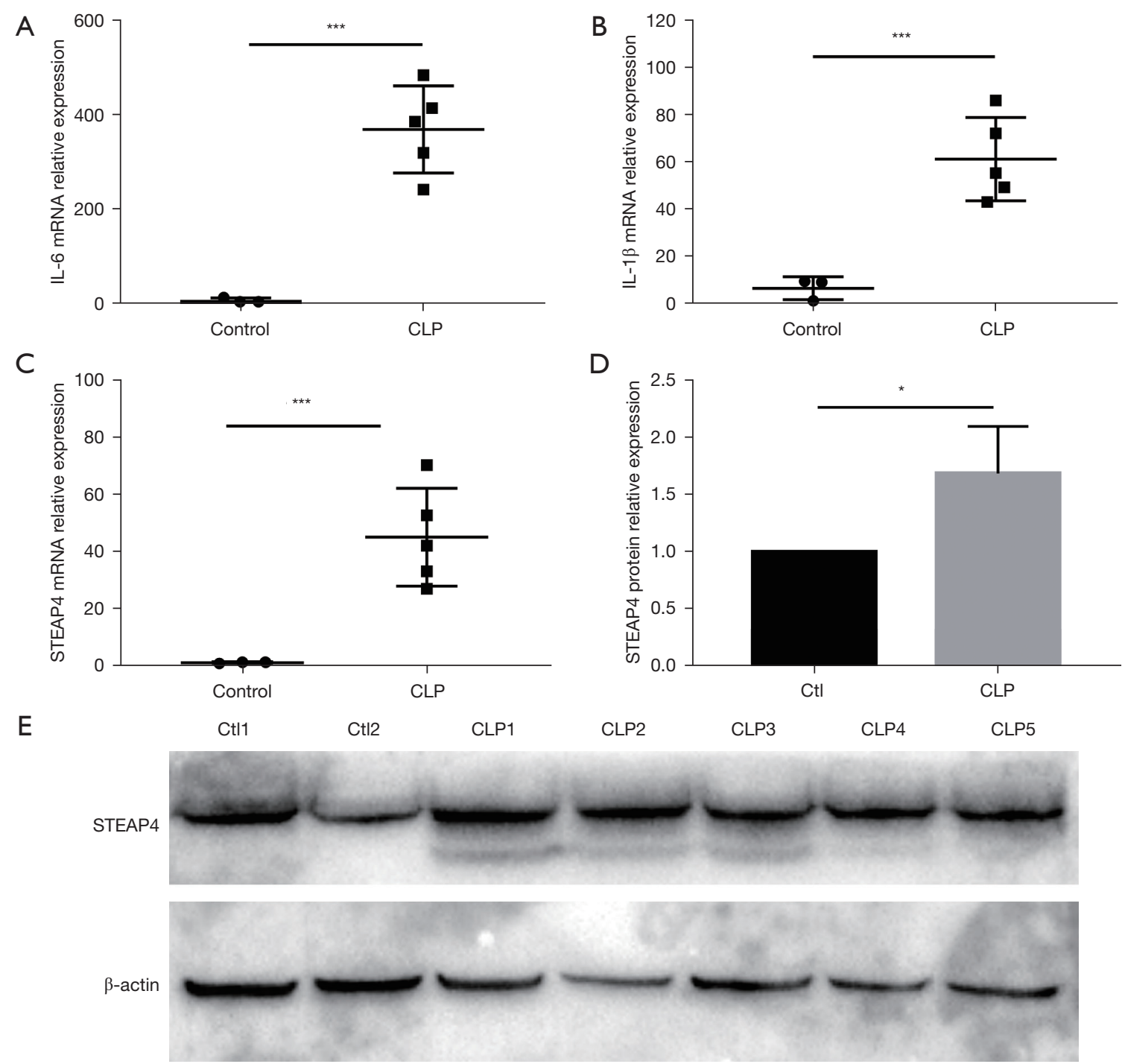

Figure 8 Expression of STEAP4 in CLP mouse liver. (A-C) The expression levels of IL-6, IL-1 $\beta$, and STEAP4 were significantly different in the livers of untreated mice and mice after the CLP operation detected by qRT-PCR; (D,E) STEAP4 protein expression was significantly different in the livers of untreated and CLP mice detected by WB. *, $\mathrm{P}<0.05$; ${ }^{* *}, \mathrm{P}<0.01$. Ctl, control; CLP, cecal ligation and perforation; STEAP4, six-transmembrane epithelial antigen of the prostate 4.

\section{Acknowledgments}

The authors thank The Affiliated Hospital of Nantong University for expert technical assistance and statistical analysis support.

Funding: This research received funding from the National Natural Science Foundation of China Youth Fund (81801893), the Nantong Clinical Medicine Research Center (HS2019005), Natural Science Research Project of Nantong Science and Technology Bureau (MS12021021, MS12020006, MS12020017, MS12021035), Special Fund for Emergency Medical Research of Ruiyi of Peking Union Medical College Hospital (R2019023), Jiangsu Planned Projects for Postdoctoral Research Fund (2021K031A), Deputy General Manager Project of Science and Technology of Jiangsu Province (FZ20210652), Jiangsu geriatric clinical technology application research project (LR2021047).

\section{Footnote}

Reporting Checklist: The authors have completed the 
STARD reporting checklist. Available at https://dx.doi. org/10.21037/atm-21-2794

Data Sharing Statement: Available at https://dx.doi. org/10.21037/atm-21-2794

Conflicts of Interest: All authors have completed the ICMJE uniform disclosure form (available at https://dx.doi. org/10.21037/atm-21-2794). The authors have no conflicts of interest to declare.

Ethical Statement: The authors are accountable for all aspects of the work in ensuring that questions related to the accuracy or integrity of any part of the work are appropriately investigated and resolved. All procedures performed in this study involving human participants were in accordance with the Declaration of Helsinki (as revised in 2013). The study was approved by Ethics Committee of the Affiliated Hospital of Nantong University (No. 2017-L021) and informed consent was taken from all the patients. All animal experiments were undertaken in accordance with the National Institute of Health Guide for the Care and Use of Laboratory Animals. Animal experiments were approved by Ethics Committee of Affiliated Hospital of Nantong University.

Open Access Statement: This is an Open Access article distributed in accordance with the Creative Commons Attribution-NonCommercial-NoDerivs 4.0 International License (CC BY-NC-ND 4.0), which permits the noncommercial replication and distribution of the article with the strict proviso that no changes or edits are made and the original work is properly cited (including links to both the formal publication through the relevant DOI and the license). See: https://creativecommons.org/licenses/by-nc-nd/4.0/.

\section{References}

1. Singer M, Deutschman CS, Seymour CW, et al. The Third International Consensus Definitions for Sepsis and Septic Shock (Sepsis-3). JAMA 2016;315:801-10.

2. Cecconi M, Evans L, Levy M, et al. Sepsis and septic shock. Lancet 2018;392:75-87.

3. Pool R, Gomez H, Kellum JA. Mechanisms of Organ Dysfunction in Sepsis. Crit Care Clin 2018;34:63-80.

4. Scarl RT, Lawrence CM, Gordon HM, et al. STEAP4: its emerging role in metabolism and homeostasis of cellular iron and copper. J Endocrinol 2017;234:R123-R134.
5. Ohgami RS, Campagna DR, McDonald A, et al. The Steap proteins are metalloreductases. Blood 2006;108:1388-94.

6. Moldes M, Lasnier F, Gauthereau X, et al. Tumor necrosis factor-alpha-induced adipose-related protein (TIARP), a cell-surface protein that is highly induced by tumor necrosis factor-alpha and adipose conversion. J Biol Chem 2001;276:33938-46.

7. Chen X, Zhu C, Ji C, et al. STEAP4, a gene associated with insulin sensitivity, is regulated by several adipokines in human adipocytes. Int J Mol Med 2010;25:361-7.

8. Cheng R, Qiu J, Zhou XY, et al. Knockdown of STEAP4 inhibits insulin-stimulated glucose transport and GLUT4 translocation via attenuated phosphorylation of Akt, independent of the effects of EEA1. Mol Med Rep 2011;4:519-23.

9. Gordon HM, Majithia N, MacDonald PE, et al. STEAP4 expression in human islets is associated with differences in body mass index, sex, HbAlc, and inflammation. Endocrine 2017;56:528-37.

10. Wang SB, Lei T, Zhou LL, et al. Functional analysis and transcriptional regulation of porcine six transmembrane epithelial antigen of prostate 4 (STEAP4) gene and its novel variant in hepatocytes. Int J Biochem Cell Biol 2013;45:612-20.

11. Chuang CT, Guh JY, Lu CY, et al. Steap4 attenuates high glucose and S100B-induced effects in mesangial cells. J Cell Mol Med 2015;19:1234-44.

12. Tanaka Y, Matsumoto I, Iwanami K, et al. Sixtransmembrane epithelial antigen of prostate4 (STEAP4) is a tumor necrosis factor alpha-induced protein that regulates IL-6, IL-8, and cell proliferation in synovium from patients with rheumatoid arthritis. Mod Rheumatol 2012;22:128-36.

13. Rittirsch D, Huber-Lang MS, Flierl MA, et al. Immunodesign of experimental sepsis by cecal ligation and puncture. Nat Protoc 2009;4:31-6.

14. Li A, Li J, Bao Y, Yuan D, et al. Xuebijing injection alleviates cytokine-induced inflammatory liver injury in CLP-induced septic rats through induction of suppressor of cytokine signaling 1. Experimental and therapeutic medicine 2016;12:1531-36.

15. Tanaka Y, Matsumoto I, Iwanami K, et al. Sixtransmembrane epithelial antigen of prostate 4 (STEAP4) is expressed on monocytes/neutrophils, and is regulated by TNF antagonist in patients with rheumatoid arthritis. Clinical and experimental rheumatology 2012;30:99-102.

16. Liang Y, Xing X, Beamer M, et al. Six-transmembrane epithelial antigens of the prostate comprise a novel 


\section{Page 12 of 12}

inflammatory nexus in patients with pustular skin disorders. J Allergy Clin Immunol 2017;139:1217-27.

17. Ozmen F, Ozmen M, Gelecek S, et al. STEAP4 and HIF$1 \alpha$ gene expressions in visceral and subcutaneous adipose tissue of the morbidly obese patients. Mol Immunol 2016;73:53-9.

18. Liao Y, Zhao J, Bulek K, et al. Inflammation mobilizes copper metabolism to promote colon tumorigenesis via an IL-17-STEAP4-XIAP axis. Nat Commun 2020;11:900.

19. Qin DN, Kou CZ, Ni YH, et al. Monoclonal antibody to the six-transmembrane epithelial antigen of prostate 4 promotes apoptosis and inhibits proliferation and glucose uptake in human adipocytes. Int $\mathrm{J}$ Mol Med 2010;26:803-11.

20. Xia B, Meng Q, Feng X, et al. Probing the molecular regulation of lipopolysaccharide stress in piglet liver

Cite this article as: Jiang $\mathrm{H}$, Dong $\mathrm{Y}$, Yan D, Wu Y, Wang Y, Ren Y, Mao G, Liang G, Liu W, Zhou Y, Huang Z, Qi L. The expression of STEAP4 in peripheral blood predicts the outcome of septic patients. Ann Transl Med 2021;9(20):1519. doi: $10.21037 /$ atm-21-2794

\section{Jiang et al. STEAP4 predicts the outcome of septic patients.}

by comparative proteomics analysis. Electrophoresis 2018;39:2321-31.

21. Jiang C, Wu B, Xue M, et al. Inflammation accelerates copper-mediated cytotoxicity through induction of sixtransmembrane epithelial antigens of prostate 4 expression. Immunol Cell Biol 2021;99:392-402.

22. Xue X, Bredell BX, Anderson ER, et al. Quantitative proteomics identifies STEAP4 as a critical regulator of mitochondrial dysfunction linking inflammation and colon cancer. Proc Natl Acad Sci U S A 2017;114:E9608-E9617.

23. Uhle F, Lichtenstern C, Brenner T, et al. Pathophysiology of sepsis. Anasthesiol Intensivmed Notfallmed Schmerzther 2015;50:114-22.

(English Language Editor: C. Betlazar-Maseh) 